\title{
Detailed spectral and morphological analysis of the shell type supernova remnant RCW 86
}

H.E.S.S. Collaboration, A. Abramowski ${ }^{1}$, F. Aharonian ${ }^{2,3,4}$, F. Ait Benkhali ${ }^{2}$, A. G. Akhperjanian ${ }^{5,4}$, E. O. Angüner ${ }^{6}$, M. Backes ${ }^{7}$, A. Balzer ${ }^{8}$, Y. Becherini ${ }^{9}$, J. Becker Tjus ${ }^{10}$, D. Berge ${ }^{11}$, S. Bernhard ${ }^{12}$, K. Bernlöhr ${ }^{2}$, E. Birsin ${ }^{6}$, R. Blackwell ${ }^{13}$, M. Böttcher ${ }^{14}$, C. Boisson $^{15}$, J. Bolmont ${ }^{16}$, P. Bordas ${ }^{2}$, J. Bregeon ${ }^{17}$, F. Brun ${ }^{18}$, P. Brun ${ }^{18}$, M. Bryan ${ }^{8}$, T. Bulik ${ }^{19}$, J. Carr ${ }^{20}$, S. Casanova ${ }^{21,2}$, N. Chakraborty ${ }^{2}$, R. Chalme-Calvet ${ }^{16}$, R. C. G. Chaves ${ }^{17,22}$, A. Chen ${ }^{23}$, J. Chevalier ${ }^{24}$, M. Chrétien ${ }^{16}$, S. Colafrancesco ${ }^{23}$, G. Cologna ${ }^{25}$, B. Condon ${ }^{26}$, J. Conrad ${ }^{27,28}$, C. Couturier ${ }^{16}$, Y. Cui ${ }^{29}$, I. D. Davids ${ }^{14,7}$, B. Degrange ${ }^{30}$, C. Deil ${ }^{2}$, P. deWilt ${ }^{13}$, A. Djannati-Atai ${ }^{31}$, W. Domainko ${ }^{2}$, A. Donath ${ }^{2}$, L. O'C. Drury ${ }^{3}$, G. Dubus ${ }^{32}$, K. Dutson ${ }^{33}$, J. Dyks ${ }^{34}$, M. Dyrda ${ }^{21}$, T. Edwards ${ }^{2}$, K. Egberts ${ }^{35}$, P. Eger $^{2}$, J.-P. Ernenwein ${ }^{20}$, P. Espigat ${ }^{31}$, C. Farnier ${ }^{27}$, S. Fegan ${ }^{30}$, F. Feinstein ${ }^{17}$, M. V. Fernandes ${ }^{1}$, D. Fernandez ${ }^{17}$, A. Fiasson ${ }^{24}$, G. Fontaine ${ }^{30}$, A. Förster ${ }^{2}$, M. Füßling ${ }^{36}$, S. Gabici ${ }^{31}$, M. Gajdus ${ }^{6}$, Y. A. Gallant ${ }^{17}$,

T. Garrigoux ${ }^{16}$, G. Giavitto ${ }^{36}$, B. Giebels ${ }^{30}$, J. F. Glicenstein ${ }^{18}$, D. Gottschall ${ }^{29}$, A. Goyal ${ }^{37}$, M.-H. Grondin ${ }^{26}$, M. Grudzińska ${ }^{19}$, D. Hadasch ${ }^{12}$, S. Häffner ${ }^{38}$, J. Hahn ${ }^{2}$, J. Hawkes ${ }^{13}$, G. Heinzelmann ${ }^{1}$, G. Henri' ${ }^{32}$, G. Hermann ${ }^{2}$, O. Hervet ${ }^{15}$, A. Hillert ${ }^{2}$, J. A. Hinton ${ }^{2,33}$, W. Hofmann ${ }^{2}$, P. Hofverberg ${ }^{2}$, C. Hoischen ${ }^{35}$, M. Holler ${ }^{30}$, D. Horns ${ }^{1}$, A. Ivascenko ${ }^{14}$, A. Jacholkowska ${ }^{16}$, M. Jamrozy ${ }^{37}$, M. Janiak ${ }^{34}$, F. Jankowsky ${ }^{25}$,

I. Jung-Richardt ${ }^{38,{ }^{*}}$, M. A. Kastendieck ${ }^{1}$, K. Katarzyński ${ }^{39}$, U. Katz ${ }^{38}$, D. Kerszberg ${ }^{16}$, B. Khélifi ${ }^{31}$, M. Kieffer ${ }^{16}$, S. Klepser ${ }^{36}$, D. Klochkov ${ }^{29}$, W. Kluźniak ${ }^{34}$, D. Kolitzus ${ }^{12}$, Nu. Komin ${ }^{23}$, K. Kosack ${ }^{18}$, S. Krakau ${ }^{10}$, F. Krayzel ${ }^{24}$, P. P. Krüger ${ }^{14}$, H. Laffon ${ }^{26}$, G. Lamanna ${ }^{24}$, J. Lau ${ }^{13}$,

J. Lefaucheur ${ }^{31}$, V. Lefranc ${ }^{18}$, A. Lemière ${ }^{31}$, M. Lemoine-Goumard ${ }^{26}$, J.-P. Lenain ${ }^{16}$, T. Lohse ${ }^{6}$, A. Lopatin ${ }^{38}$, M. Lorentz ${ }^{18}$, C.-C. Lu ${ }^{2}$, R. Lui ${ }^{2}$,

V. Marandon ${ }^{2}$, A. Marcowith ${ }^{17}$, C. Mariaud ${ }^{30}$, R. Marx ${ }^{2}$, G. Maurin ${ }^{24}$, N. Maxted ${ }^{17}$, M. Mayer ${ }^{6}$, P. J. Meintjes ${ }^{40}$, U. Menzler ${ }^{10}$, M. Meyer ${ }^{27}$,

A. M. W. Mitchell ${ }^{2}$, R. Moderski ${ }^{34}$, M. Mohamed ${ }^{25}$, K. Morå27 ${ }^{27}$ E. Moulin ${ }^{18}$, T. Murach ${ }^{6}$, M. de Naurois ${ }^{30}$, J. Niemiec ${ }^{21}$, L. Oakes $^{6}$, H. Odaka $^{2}$,

S. Öttl ${ }^{12}$, S. Ohm ${ }^{36}$, B. Opitz ${ }^{1}$, M. Ostrowski ${ }^{37}$, I. Oya ${ }^{36}$, M. Panter ${ }^{2}$, R. D. Parsons ${ }^{2}$, M. Paz Arribas ${ }^{6}$, N. W. Pekeur ${ }^{14}$, G. Pelletier $^{32}$,

P.-O. Petrucci ${ }^{32}$, B. Peyaud ${ }^{18}$, S. Pita ${ }^{31}$, H. Poon ${ }^{2}$, D. Prokhorov ${ }^{9}$, H. Prokoph ${ }^{9}$, G. Pühlhofer ${ }^{29}$, M. Punch ${ }^{31}$, A. Quirrenbach $^{25}$, S. Raab ${ }^{38}$,

I. Reichardt ${ }^{31}$, A. Reimer ${ }^{12}$, O. Reimer ${ }^{12}$, M. Renaud ${ }^{17}$, R. de los Reyes ${ }^{2}$, F. Rieger ${ }^{2,41}$, C. Romoli ${ }^{3}$, S. Rosier-Lees ${ }^{24}$, G. Rowell $^{13}$, B. Rudak $^{34}$, C. B. Rulten ${ }^{15}$, V. Sahakian ${ }^{5,4}$, D. Salek ${ }^{42}$, D. A. Sanchez ${ }^{24}$, A. Santangelo ${ }^{29}$, M. Sasaki ${ }^{29}$, R. Schlickeiser ${ }^{10}$, F. Schüssler ${ }^{18}$, A. Schulz ${ }^{36}$,

U. Schwanke ${ }^{6}$, S. Schwemmer ${ }^{25}$, A. S. Seyffert ${ }^{14}$, R. Simoni ${ }^{8}$, H. Sol ${ }^{15}$, F. Spanier ${ }^{14}$, G. Spengler ${ }^{27}$, F. Spies ${ }^{1}$, Ł. Stawarz $^{37}$, R. Steenkamp ${ }^{7}$, C. Stegmann ${ }^{35,36}$, F. Stinzing ${ }^{38}$, K. Stycz ${ }^{36}$, I. Sushch ${ }^{14}$, J.-P. Tavernet ${ }^{16}$, T. Tavernier ${ }^{31}$, A. M. Taylor ${ }^{3}$, R. Terrier ${ }^{31}$, M. Tluczykont $^{1}$,

C. Trichard ${ }^{24}$, R. Tuffs ${ }^{2}$, K. Valerius ${ }^{38}$, J. van der Walt ${ }^{14}$, C. van Eldik ${ }^{38}$, B. van Soelen ${ }^{40}$, G. Vasileiadis ${ }^{17}$, J. Veh $^{38}$, C. Venter $^{14}$, A. Viana $^{2}$,

P. Vincent ${ }^{16}$, J. Vink ${ }^{8}$, F. Voisin ${ }^{13}$, H. J. Völk ${ }^{2}$, T. Vuillaume ${ }^{32}$, S. J. Wagner ${ }^{25}$, P. Wagner ${ }^{6}$, R. M. Wagner ${ }^{27}$, M. Weidinger ${ }^{10}$, R. White $^{33,2}$,

A. Wierzcholska ${ }^{25,21}$, P. Willmann ${ }^{38}$, A. Wörnlein ${ }^{38}$, D. Wouters ${ }^{18}$, R. Yang ${ }^{2}$, V. Zabalza ${ }^{33}$, D. Zaborov ${ }^{30}$, M. Zacharias ${ }^{25}$, A. A. Zdziarski $^{34}$,

$$
\text { A. Zech }{ }^{15}, \text { F. Zefi }{ }^{30} \text {, and N. Żywucka }{ }^{37}
$$

(Affiliations can be found after the references)

Received 18 May 2015 / Accepted 9 November 2015

\section{ABSTRACT}

Aims. We aim for an understanding of the morphological and spectral properties of the supernova remnant RCW 86 and for insights into the production mechanism leading to the RCW 86 very high-energy $\gamma$-ray emission.

Methods. We analyzed High Energy Spectroscopic System (H.E.S.S.) data that had increased sensitivity compared to the observations presented in the RCW 86 H.E.S.S. discovery publication. Studies of the morphological correlation between the $0.5-1 \mathrm{keV} \mathrm{X-ray} \mathrm{band,} \mathrm{the} 2-5 \mathrm{keV}$ X-ray band, radio, and $\gamma$-ray emissions have been performed as well as broadband modeling of the spectral energy distribution with two different emission models.

Results. We present the first conclusive evidence that the $\mathrm{TeV} \gamma$-ray emission region is shell-like based on our morphological studies. The comparison with $2-5 \mathrm{keV}$ X-ray data reveals a correlation with the $0.4-50 \mathrm{TeV} \gamma$-ray emission. The spectrum of RCW 86 is best described by a power law with an exponential cutoff at $E_{\text {cut }}=\left(3.5 \pm 1.2_{\text {stat }}\right) \mathrm{TeV}$ and a spectral index of $\Gamma \approx 1.6 \pm 0.2$. A static leptonic one-zone model adequately describes the measured spectral energy distribution of RCW 86, with the resultant total kinetic energy of the electrons above $1 \mathrm{GeV}$ being equivalent to $\sim 0.1 \%$ of the initial kinetic energy of a Type Ia supernova explosion $\left(10^{51} \mathrm{erg}\right)$. When using a hadronic model, a magnetic field of $B \approx 100 \mu \mathrm{G}$ is needed to represent the measured data. Although this is comparable to formerly published estimates, a standard $\mathrm{E}^{-2}$ spectrum for the proton distribution cannot describe the $\gamma$-ray data. Instead, a spectral index of $\Gamma_{\mathrm{p}} \approx 1.7$ would be required, which implies that $\sim 7 \times 10^{49} / n_{\mathrm{cm}}{ }^{-3} \mathrm{erg}$ has been transferred into high-energy protons with the effective density $n_{\mathrm{cm}^{-3}}=n / 1 \mathrm{~cm}^{-3}$. This is about $10 \%$ of the kinetic energy of a typical Type Ia supernova under the assumption of a density of $1 \mathrm{~cm}^{-3}$.

Key words. astroparticle physics - gamma rays: general - ISM: supernova remnants - cosmic rays

\section{Introduction}

Supernova remnants (SNR) are prime candidates to be sources of Galactic cosmic rays. The detection of very-high-energy (VHE)

\footnotetext{
* Corresponding author: H.E.S.S. Collaboration, e-mail: contact.hess@hess-experiment.eu
}

$\gamma$-ray $(E>100 \mathrm{GeV})$ and nonthermal X-ray emission from SNRs has shown that they do in fact accelerate particles to energies above $100 \mathrm{TeV}$ (see, e.g., Hinton \& Hofmann 2009; Aharonian 2013).

RCW 86 (also known as G315.4-2.3 or MSH 14-63) is located at a distance of $(2.5 \pm 0.5) \mathrm{kpc}$ (Helder et al. 2013). It is 
almost circular in shape with a diameter of about 40' clearly showing a shell-like structure in the optical (Smith 1997), radio (Kesteven \& Caswell 1987), infrared (Williams et al. 2011) and X-ray (Pisarski et al. 1984) regimes. The source has been detected in $\gamma$-rays (Aharonian et al. 2009; Yuan et al. 2014), but a shell-like structure was not resolved. RCW 86 is a young SNR, which is about 1800 years old, based on the probable connection to the historical supernova SN 185 recorded by Chinese astronomers in 185 AD (Stephenson \& Green 2002; Smith 1997; Dickel et al. 2001; Vink et al. 2006). The nature of the RCW 86 supernova $(\mathrm{SN})$ progenitor was under intense discussion in the context of its young age and its unusually large size with a radius of $R \approx 15 d_{2.5} \mathrm{pc}\left(d_{2.5}\right.$ : the distance to RCW 86 in units of $2.5 \mathrm{kpc}$ ). Williams et al. (2011) comprehensively studied all arguments about the type of the progenitor of RCW 86 and conducted hydrodynamic simulations to explain the observed characteristics. They concluded that RCW 86 is likely the remnant of a Type Ia supernova. The explosion was off-center in a low-density cavity carved by the progenitor system (see also Vink et al. 1997; Broersen et al. 2014).

The physical conditions vary within the volume of RCW 86. While slow shocks have been measured $\left(\sim 600-800 \mathrm{~km} \mathrm{~s}^{-1}\right.$; Long \& Blair 1990; Ghavamian et al. 2001) in the southwest (SW) and northwest (NW) regions along with relatively high post-shock gas densities ( $2 \mathrm{~cm}^{-3}$; Williams et al. 2011), X-ray measurements by Helder et al. (2009) indicate high velocities of $6000 \pm 2800 \mathrm{~km} \mathrm{~s}^{-1}$ in the northeast (NE) whereas optical measurements of specific knots in this region by Helder et al. (2013) show large variations in proper motions between 700-2000 $\mathrm{km} \mathrm{s}^{-1}$ and low densities of $\sim 0.1-0.3 \mathrm{~cm}^{-3}$ (see Yamaguchi et al. 2011). In the SW, NE, and to a lesser extent in the NW region, nonthermal X-ray emission is detected (Bamba et al. 2000; Vink et al. 1997, 2006; Williams et al. 2011). Near a region of Fe-K-line emission in the SW, there is nonthermal emission possibly synchrotron radiation from electrons accelerated at the reverse shock (Rho et al. 2002).

Here, we present a new analysis of VHE $\gamma$-ray data of RCW 86 taken with the High Energy Stereoscopic System (H.E.S.S.). When the discovery of RCW 86 was published by the H.E.S.S. Collaboration, the morphology in the $\mathrm{TeV}$ $\gamma$-ray regime could not be resolved owing to limited statistics (Aharonian et al. 2009). The new analysis benefits from significantly improved statistics and addresses whether RCW 86 has a shell structure in the VHE $\gamma$-ray regime. In addition, the larger data set allows for a broadband modeling of the spectral energy distribution with both a leptonic and an hadronic one-zone model. We restricted ourselves to two simple models since it is unreasonable to use more intricate models with additional parameters that cannot be determined owing to limited statistics.

\section{H.E.S.S. observations and analysis method}

The H.E.S.S. is located in the Khomas Highland of Namibia at a height of about $1800 \mathrm{~m}$ above sea level. In its first phase, the system consisted of four identical imaging Cherenkov telescopes, each with a mirror area of $107 \mathrm{~m}^{2}$ and a large field of view of $5^{\circ}$ (Bernlöhr et al. 2003). The data presented in this paper were taken during this phase.

In 2009 the H.E.S.S. Collaboration announced the discovery of RCW 86 in the VHE regime (Aharonian et al. 2009). The source morphology of RCW 86 was thoroughly studied, but the existing data did not suffice to settle the question of whether the VHE emission originates from a shell or from a spherical region. Between 2007 and 2011, observations of the neighboring source

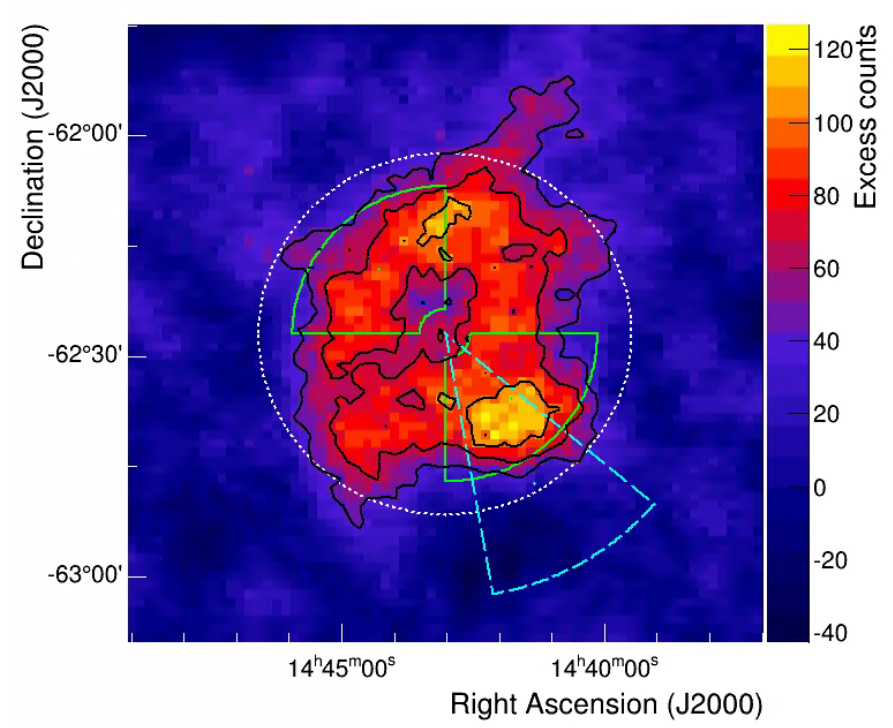

Fig. 1. VHE $\gamma$-ray emission of RCW 86. The sky map is extracted with faint analysis cuts and smoothed with a Gaussian filter with $\sigma_{\text {smooth }}=$ 0.06 to reduce the effect of statistical fluctuations. Black contours correspond to $3,5,7 \sigma$ significance. The white dotted circle depicts the integration region chosen for the spectral analysis. The dashed cyan sector shows the position angle range of the radial profile in Fig. 3. The two green sectors (solid lines) give the extraction regions for spectra of the SW and NE regions discussed in Sect. 3.

H.E.S.S. J1458-608 (de Los Reyes Lopez 2011) and the scan of the Galactic plane have added additional observation time to the data set, which now amounts to $\sim 57 \mathrm{~h}$. The zenith angles of these observations range from $36^{\circ}$ to $53^{\circ}$ and have a mean value of $40^{\circ}$. The data were recorded with pointing offsets between $0.5^{\circ}$ and $2.5^{\circ}$, average $1.1^{\circ}$, from the RCW 86 position. The sensitivity and angular resolution were improved compared to the former publication (Aharonian et al. 2009), using an advanced analysis method (for further detail, see de Naurois \& Rolland 2009) instead of the formerly used standard Hillas-parameterbased analysis. Standard cuts were used for spectral analysis and faint source cuts for morphological analysis. The faint source cuts have an improved angular resolution at the expense of lower statistics and a higher energy threshold of about $100 \mathrm{GeV}$ (for the cut definition, see de Naurois \& Rolland 2009). The results we present are consistent with the results of a multivariate analysis technique (Ohm et al. 2009), using an independent calibration scheme.

\section{Results}

The $\gamma$-ray excess map, using the ring background subtraction technique (Berge et al. 2007), is shown in Fig. 1. The map was smoothed with a Gaussian filter to reduce the effect of statistical fluctuations. The $68 \%$ containment radius of 0.061 of the H.E.S.S. point spread function (PSF) was chosen as smoothing radius.

Since this is larger than the bin size (0.02), the pixels are correlated with each other. This is accounted for in the spatial-fitting process that we present. Extended $\gamma$-ray emission was found. We detected $1220 \pm 87$ excess $\gamma$-rays within a circular region centered at $\alpha_{\mathrm{J} 2000}=14^{\mathrm{h}} 43^{\mathrm{m}} 2.16^{\mathrm{s}}, \delta_{\mathrm{J} 2000}=-62^{\circ} 26^{\prime} 56^{\prime \prime}$ with a radius of 0.41 (see Fig. 1) and a source detection at a statistical 


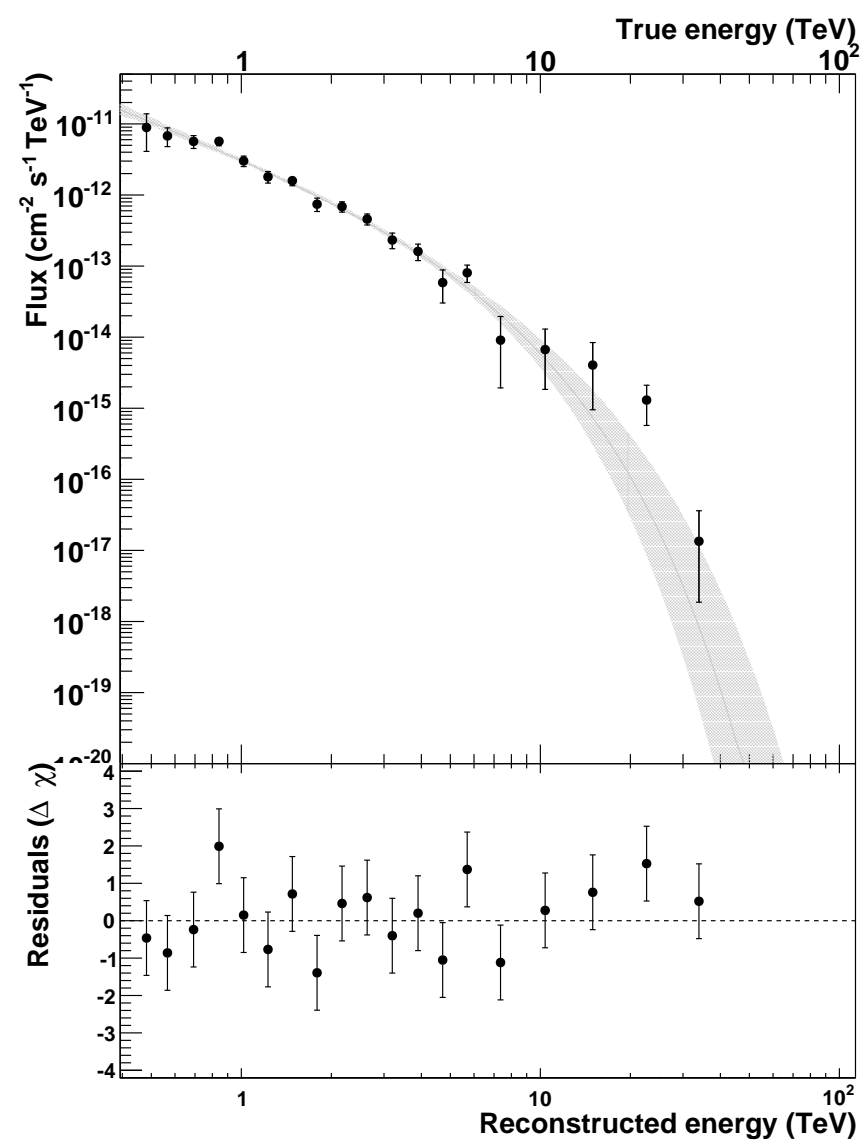

Fig. 2. Upper panel: differential energy spectrum of RCW 86 with the best-fit exponential cutoff power-law model. The error bars denote $1 \sigma$ statistical errors. The shaded area represents the $1 \sigma$ confidence level of the fitted spectrum. Bottom panel: corresponding residuals.

significance of $18.3 \sigma$. The center is given by the flux averaged centroid, which was determined with the spectral results of the previous H.E.S.S. publication of RCW 86 (Aharonian et al. 2009). The radius was adjusted to the improved analysis method and better angular resolution.

\subsection{Spectral analysis}

A fit of a power-law function $\mathrm{d} N / \mathrm{d} E=\Phi_{0}(E / 1 \mathrm{TeV})^{-\Gamma}$ to the spectral data analyzed with the standard analysis cuts yields $\Gamma=2.3 \pm 0.1_{\text {stat }} \pm 0.2_{\text {sys }}$, and the differential flux normalization at $1 \mathrm{TeV}$ of $\Phi_{0}=\left(2.9 \pm 0.2_{\text {stat }} \pm 0.6_{\text {sys }}\right) \times$ $10^{-12} \mathrm{~cm}^{-2} \mathrm{~s}^{-1} \mathrm{TeV}^{-1}$ (Log(likelihood) $=-54$; see Fig. 2). We tested if a power law with an exponential cutoff $\mathrm{d} N / \mathrm{d} E=$ $\Phi_{0}(E / 1 \mathrm{TeV})^{-\Gamma} \exp \left(-E / E_{\text {cut }}\right)$ describes the data better than a simple power law. The fit gave $\Phi(1 \mathrm{TeV})=\left(3.0 \pm 0.2_{\text {stat }} \pm 0.6_{\text {sys }}\right) \times$ $10^{-12} \mathrm{~cm}^{-2} \mathrm{~s}^{-1} \mathrm{TeV}^{-1}$ with $\Gamma=1.6 \pm 0.2_{\text {stat }} \pm 0.2_{\text {sys }}$ and a cutoff energy $E_{\text {cut }}=\left(3.5 \pm 1.2_{\text {stat }} \pm 2.2_{\text {sys }}\right) \mathrm{TeV}(\log ($ likelihood $)=$ -47.7 ), which is favored over the simple power law with a significance of $3.5 \sigma$, according to a log-likelihood ratio test. The spectral result of the power-law fit is compatible with a previous H.E.S.S. publication (Aharonian et al. 2009).

\subsection{Morphology}

To settle the question of whether the $\mathrm{TeV} \gamma$-ray emission is shell-like as found in the optical, radio, and X-ray bands, a two- dimensional log-likelihood fit was applied to the uncorrelated sky maps taking the morphological model and instrument response into account. To distinguish between different morphological structures, we used two alternative models: a sphere and a shell model. The models are obtained by line-of-sight integrals of a three-dimensional emitting sphere or shell. In the case of the shell model, the free parameters are the center position and the inner and outer radius. The sphere model is derived from the shell model by fixing the inner radius to zero. Both models have uniform emissivity. Table 1 summarizes the fit results. The shell structure is favored over the sphere structure with a significance of about $8 \sigma$. In Fig. 3 (left panel), the radial profile of the $\mathrm{TeV}$ emission itself is given together with the fit results of both models.

To facilitate the comparison, radial excess profiles of VHE $\gamma$-ray, X-ray, and radio emission are presented in the center and right panels of Fig. 3. The X-ray data is split into two energy bands: $0.5-1 \mathrm{keV}$ and $2-5 \mathrm{keV}$. The latter exhibits a higher amount of nonthermal emission in comparison to the low-energy band. The nonthermal emission in RCW 86 is not evenly distributed. Broersen et al. (2014) have shown that the NE region is dominated by nonthermal emission, while the SW region exhibits similar amounts of hot thermal and nonthermal emission. In the NW and SE regions, however, thermal emission prevails. In Fig. 3, the central panel depicts the radial profile of the whole SNR, whereas in the right panel only the data of the SW region within the position angle range ${ }^{1}$ of $190^{\circ}$ to $230^{\circ}$ were used to determine the profiles. The radio data are from Molonglo Observatory Synthesis Telescope (MOST; Whiteoak \& Green 1996) and the X-ray data are taken with the XMM-Newton X-ray telescope (Broersen et al. 2014). The radial profile of the whole remnant (Fig. 3, center panel) clearly shows that the $\mathrm{TeV}$ and the X-ray emission of the energy range between $2-5 \mathrm{keV}$ are correlated, whereas in the case of low energetic X-ray emission $(0.5-1 \mathrm{keV})$ and radio emission a weaker luminosity is found in the central region and the shell-like emission is more pronounced. The radial profiles of the SW region of $\mathrm{TeV} \gamma$-ray emission, low- and highenergy X-ray radiation (Fig. 3, right panel) again seem to reveal a correlation between the $2-5 \mathrm{keV}$ X-ray emission and the TeVemission. In addition, it seems that the maximum of the $2-5 \mathrm{keV}$ $\mathrm{X}$-ray emission is slightly more off-center than the maximum of the $\mathrm{TeV}$ emission, but this is not significant because of the low statistics of the $\mathrm{TeV}$ data. The maxima of the low-energy X-ray $(0.5-1 \mathrm{keV})$ and radio emission are at larger radii. In summary, the TeV emission and the high-energy X-ray emission (2-5 keV) are correlated throughout the remnant, whereas radio and lowenergy X-ray emission regions are further away from the center and show lower levels of emission in the central region of the remnant.

To study the influence of the different physical conditions like shock speeds and densities on the $\gamma$-ray emission, spectra of two different subregions (SW and NE) were extracted. Figure 1 shows the regions, their position, and their size. They are both centered around the best-fit position of the 3D-shell model. A Gaussian function was fitted to the radial profile of the $\gamma$-ray data and the $1 \sigma$ interval around the mean of the fit was then taken as the minimal and maximal radii of the regions. In both regions, a detection significance of about $10 \sigma$ has been achieved. The resulting spectrum is well described by a power-law model, which was fitted to the data up to an energy of $20 \mathrm{TeV}$. It is not possible to distinguish between a simple power-law spectrum and one with an exponential cutoff

\footnotetext{
1 Position angle $0^{\circ}$ corresponds to north and $90^{\circ}$ to east.
} 

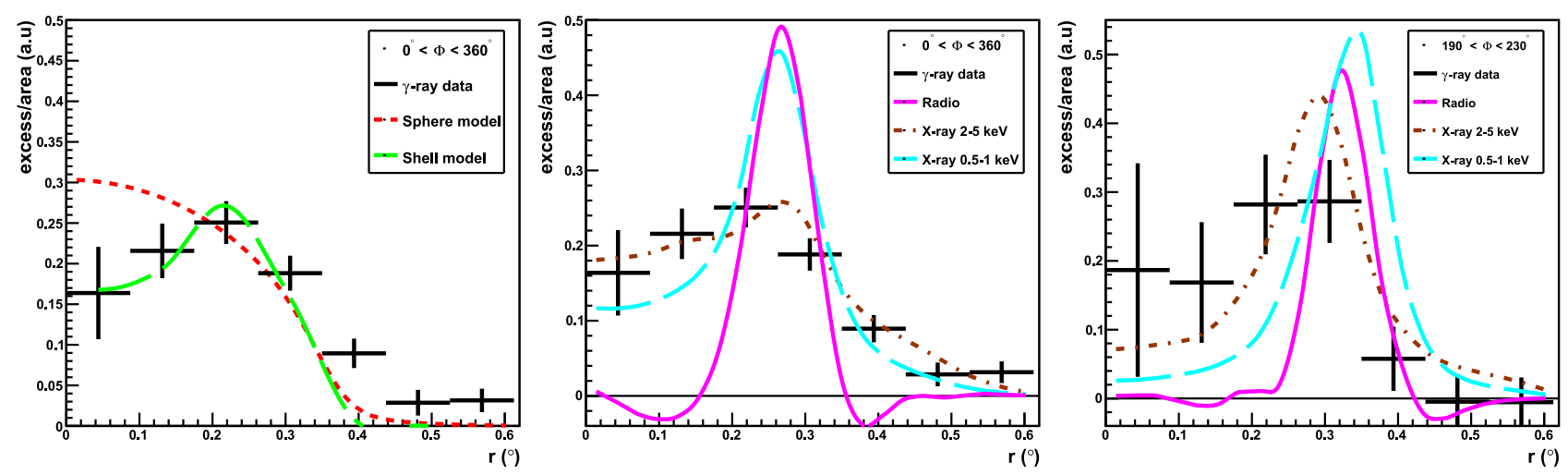

Fig. 3. Radial profiles: the shell model's best-fit center point position (see Table 1) serves as a common center for the radial profiles of the $\gamma$-ray, $\mathrm{X}$-ray, and radio data. Left panel: radial profile of the spatial distribution of the $\mathrm{TeV} \gamma$-ray emission is shown as black crosses, which depict the measured $\mathrm{TeV}$ data points and their errors. The dashed red line corresponds to the radial profile of the sphere morphology and the green line to the shell model. Center and right panels: radial profiles in the $\mathrm{TeV} \gamma$-ray, radio, $0.5-1 \mathrm{keV}$, and $2-5 \mathrm{keV} \mathrm{X}$-ray regimes for different regions of RCW 86 are shown. While the center panel covers the entire position angle range, the right panel only shows the SW region with position angles between $190^{\circ}$ and $230^{\circ}$ (position angle $0^{\circ}$ corresponds to north and $90^{\circ}$ to east; see Fig. 1). Black crosses are measured VHE excess points. The low-energy (0.5-1 keV, dashed cyan line) and high-energy (2-5 keV, dotted brown line) X-ray band data and the radio data (solid magenta line) were smoothed with the H.E.S.S.-PSF to account for the different angular resolution of the different instruments. The radio data are from Molonglo Observatory Synthesis Telescope (MOST; see Whiteoak \& Green 1996) and the X-ray data are from the XMM-Newton X-ray telescope (Broersen et al. 2014). The $\gamma$-ray and X-ray data are normalized so that the area underneath the curve is equal to one; the radio data are scaled such that the area underneath the data points is equal to 0.5.

Table 1. Results of the two-dimensional log-likelihood fit of morphological models to the H.E.S.S. data.

\begin{tabular}{cccccc}
\hline \hline Model & $\alpha_{\text {center }, 2000}$ & $\delta_{\text {center,J2000 }}$ & $r_{\text {in }}[\mathrm{pc}]$ & $r_{\text {out }}[\mathrm{pc}]$ & Log(likelihood) \\
\hline Sphere & $14^{\mathrm{h}} 42^{\mathrm{m}} 58^{\mathrm{s}} \pm 0^{\mathrm{h}} 0^{\mathrm{m}} 9^{\mathrm{s}}$ & $-62^{\circ} 25^{\prime} 48^{\prime \prime} \pm 0^{\circ} 1^{\prime} 1^{\prime \prime}$ & - & $15.9 \pm 0.6$ & -4093.35 \\
\hline Shell & $14^{\mathrm{h}} 42^{\mathrm{m}} 53^{\mathrm{s}} \pm 0^{\mathrm{h}} 0^{\mathrm{m}} 7^{\mathrm{s}}$ & $-62^{\circ} 25^{\prime} 48^{\prime \prime} \pm 0^{\circ} 1^{\prime} 48^{\prime \prime}$ & $9.5 \pm 1.4$ & $14.8 \pm 1.0$ & -4046.82 \\
\hline
\end{tabular}

Notes. $\left(\alpha_{\text {center,J2000 }}, \delta_{\text {center,J2000 }}\right)$ gives the center of the sphere and shell models. The parameters $r_{\text {in }}$ and $r_{\text {out }}$ stand for the inner and outer radius of the sphere. In the case of the sphere, $r_{\text {in }}$ is kept at zero pc.

because of the lower statistics of the quadrant data. We obtained spectral indices of $\Gamma=\left(2.5 \pm 0.2_{\text {stat }} \pm 0.2_{\text {sys }}\right)$ and of $\Gamma=\left(2.2 \pm 0.1_{\text {stat }} \pm 0.2_{\text {sys }}\right)$ as well as a differential flux normalization at $1 \mathrm{TeV}$ of $\Phi_{0}=\left(0.7 \pm 0.1_{\text {stat }} \pm 0.1_{\text {sys }}\right) \times 10^{-12} \mathrm{~cm}^{-2} \mathrm{~s}^{-1} \mathrm{TeV}^{-1}$ and $\Phi_{0}=\left(0.7 \pm 0.1_{\text {stat }} \pm 0.1_{\text {sys }}\right) \times 10^{-12} \mathrm{~cm}^{-2} \mathrm{~s}^{-1} \mathrm{TeV}^{-1}$ in the NE and SW regions, respectively. Thus, there is no significant spectral difference between both regions. The results are consistent with the spectrum of the whole remnant.

\section{Discussion}

SNRs are thought to be the primary sources for the bulk of Galactic cosmic-ray protons with energies up to $\sim 3 \mathrm{PeV}$, but the final proof is still lacking. The broadband, nonthermal emission of these sources is produced by accelerated particles through several channels, e.g., synchrotron, inverse Compton, nonthermal Bremsstrahlung, and neutral pion decay. To achieve insights into the $\gamma$-ray production taking place in SNRs, the different processes have to be disentangled and analyzed. We present the results of the broadband data of RCW 86 in two different scenarios for radiation mechanism for the $\gamma$-ray emission: inverse Compton (IC) scattering of electrons off ambient photons (leptonic scenario) or proton-proton interaction with the ambient medium (hadronic scenario). For both cases, we have modeled the broadband emission of RCW 86 with a simple static one-zone model (presented in Acero et al. 2010), where the radiation of the different wavebands is produced within the same region with a constant magnetic field. The current en- ergy distributions of the particles (electrons and/or protons) are given by a power law with an exponential cutoff. Synchrotron radiation and IC scattering on the cosmic microwave background and the local interstellar (optical and infrared) radiation fields (see Porter \& Strong 2005) were taken into account with energy densities of $0.66 \mathrm{eV} \mathrm{cm}^{-3}$ (dust) and $0.94 \mathrm{eV} \mathrm{cm}^{-3}$ (stars). In the case of the hadronic scenario, the $\gamma$-ray production was calculated following Kelner et al. (2006). Nonthermal Bremsstrahlung is neglected because of the low ambient density $\leq 1 \mathrm{~cm}^{-3}$. The modeling was done under the assumption that the distance of RCW 86 is $2500 \mathrm{pc}$, the outer radius is $15 \mathrm{pc}$, and the shell thickness is $5 \mathrm{pc}$. The emission at all wavelengths was calculated for the shell region. The stationary one-zone model is however an oversimplification because the radio morphology and the nonthermal X-ray morphology differ, which cannot be explained by this kind of model. The spectral energy distribution presented in Fig. 4 is composed of the VHE $\gamma$-ray spectra presented in Sect. 3, the Fermi-LAT data points (Yuan et al. 2014), the X-ray spectra of ASCA and RXTE (Lemoine-Goumard et al. 2012), and the radio data from the Molonglo at $408 \mathrm{MHz}$ and Parkes at $5 \mathrm{GHz}$ (Caswell et al. 1975; Lemoine-Goumard et al. 2012).

In the leptonic case, the broadband data can be described by a one-zone model using an electron spectrum with a spectral index of $\Gamma_{\mathrm{e}} \approx 2.3$, an exponential cutoff at $E_{\text {cut }} \approx 19 \mathrm{TeV}$, and a magnetic field of $B \approx 22 \mu \mathrm{G}$ (see Fig. 4). These results are comparable to those of Lemoine-Goumard et al. (2012) and Yuan et al. (2014) and the magnetic field is comparable 


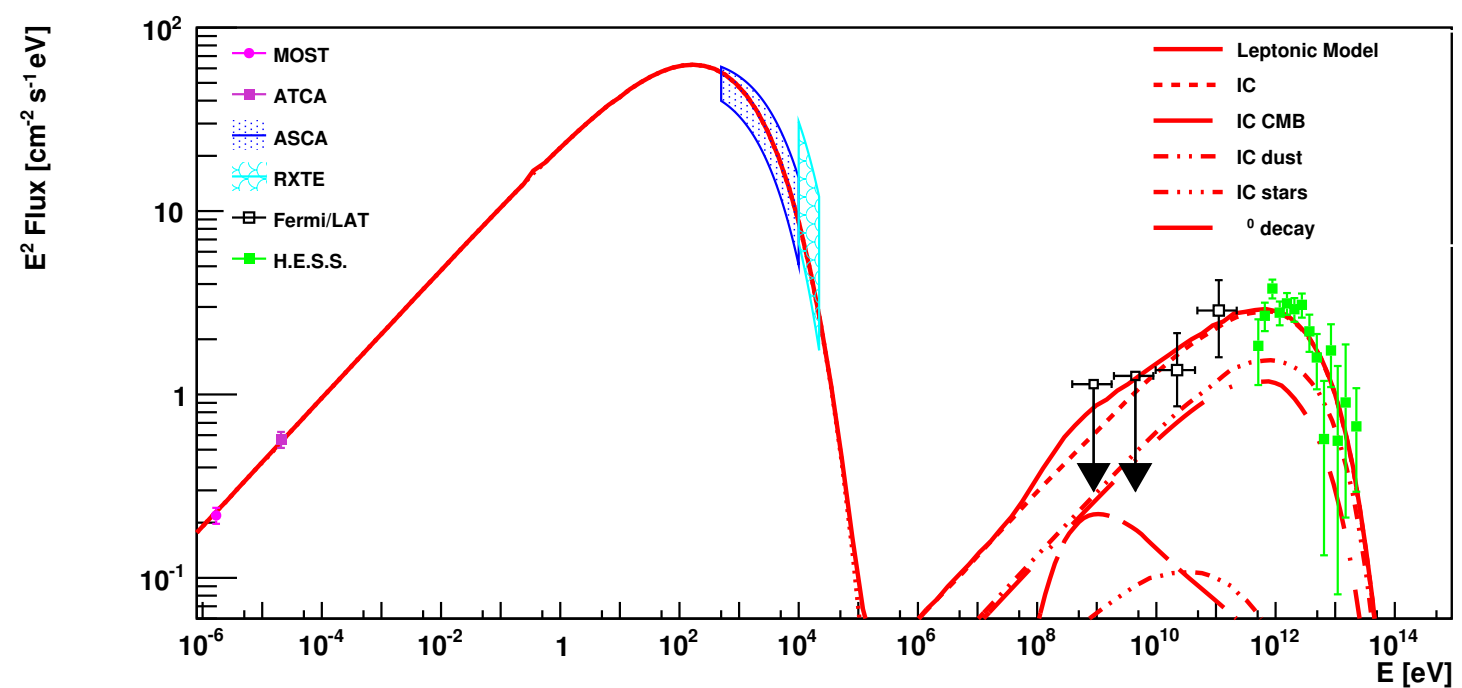

Fig. 4. Spectral energy distribution of RCW 86 for a leptonic scenario. The red solid lines denote the total broadband emission from the one-zone modeling discussed in Sect. 4. The dotted lines show the IC-contributions and the dashed line is that of the $\pi^{0}$-decay contribution. The radio data points are from Molonglo at $408 \mathrm{MHz}$ and Parkes at $5 \mathrm{GHz}$ (Caswell et al. 1975; Lemoine-Goumard et al. 2012). X-ray data are from ASCA and RXTE from Lemoine-Goumard et al. (2012). The Fermi-LAT data points are taken from Yuan et al. (2014) and the H.E.S.S. data are from this analysis.

to estimates by Vink et al. (2006). The total kinetic energy of all electrons above $1 \mathrm{GeV}$ amounts to $\sim 10^{48} \mathrm{erg}$, which is about $0.1 \%$ of the total energy of a typical Type Ia supernova $\left(10^{51} \mathrm{erg}\right)$. We obtain an upper limit for the energy injected into accelerated protons of $\sim 10^{49} n_{\mathrm{cm}^{-3}}$ erg with the effective density $n_{\mathrm{cm}^{-3}}=n / 1 \mathrm{~cm}^{-3}$ also taking into account protons with the same spectral index as the electrons and a conservative chosen energy cutoff of $100 \mathrm{TeV}$. A higher amount of energy is not compatible with the Fermi-LAT upper limits (see Fig. 4). This energy limit implies an electron-to-proton ratio above $1 \mathrm{GeV}$ of $K_{\text {ep }} \geq 10^{-1}$. These results are in good agreement with those obtained by Lemoine-Goumard et al. (2012).

In the case of the hadronic scenario, the $\gamma$-rays are produced via proton-proton interactions and subsequent neutral pion decay whereas the X-rays are still produced via synchrotron radiation of high-energetic electrons. Therefore, the electron fraction has to be lower and the magnetic field stronger. A standard proton spectrum with $\Gamma_{\mathrm{p}}=2$ and a total energy in accelerated protons at emission time of $W_{\mathrm{p}} \approx 2.1 \times 10^{50} / n_{\mathrm{cm}^{-3}} \mathrm{erg}$ would describe the H.E.S.S. measurements, but is incompatible with the Fermi-LAT results (see Fig. 5 black dashed line). This was already pointed out by Lemoine-Goumard et al. (2012). The proton spectrum, which reproduces the $\gamma$-ray data, has a spectral index of $\Gamma_{\mathrm{p}} \approx 1.7$ and a cutoff energy of $E_{\text {cut }}=35 \mathrm{TeV}$. The index lies between the spectral index of the test particle approach and the spectral index with strong modified shocks (Malkov 1999; Berezhko \& Ellison 1999). The blue line in Fig. 5 presents this hadronic model. The total energy of all protons above $1 \mathrm{GeV}$ is $W_{\mathrm{p}} \approx 9 \times 10^{49} / n_{\mathrm{cm}^{-3}} \mathrm{erg}$, which means that a fraction of $0.1 / n_{\mathrm{cm}^{-3}}$ of the supernova (Type Ia) energy has to be converted into high-energy protons. The electron-to-proton ratio is $K_{\text {ep }}=10^{-3}$. To model the radio and X-ray data, an electron spectrum with spectral index of $\Gamma_{\mathrm{e}} \approx 2.3$ and a cutoff energy of $E_{\text {cut }}=9 \mathrm{TeV}$ is needed and a magnetic field strength of $100 \mu \mathrm{G}$. This is comparable to two different estimates: one by Völk et al. (2005) of $99_{-26}^{+46} \mu \mathrm{G}$, which was deduced from the thickness of the filaments in the SW region, and another one calculated by Arbutina et al. (2012) of a volume-averaged magnetic field of $\sim 70 \mu \mathrm{G}$. The total kinetic energy of the protons above $1 \mathrm{GeV}$ was found to be $W_{\mathrm{p}} \approx 9 \times 10^{49} / n_{\mathrm{cm}^{-3}} \mathrm{erg}$, which means that about $10 \% / n_{\mathrm{cm}^{-3}}$ of the supernova (Type Ia) energy has to be converted into high-energy protons.

As already mentioned, the hadronic model cannot reproduce the radio data with an electron spectrum that has the same spectral index as the proton spectrum $\left(\Gamma_{\mathrm{p}}=1.7\right)$. This is in conflict with the expectation that electrons and protons exhibit the same dynamics at relativistic energies up to an energy where electron synchrotron losses become important.

Lemoine-Goumard et al. (2012) applied a two-zone model to the data to overcome this problem. They introduced a separate second leptonic population to explain the radio emission with the consequence that the lower limit on the magnetic field was $50 \mu \mathrm{G}$. The X-ray emission was reproduced with an injection spectrum with a power-law index of $\Gamma=1.8$, a break at $3 \mathrm{TeV}$, caused by synchrotron cooling, and an exponential cut-off at $20 \mathrm{TeV}$. The energy injected into hadrons was $\sim 7 \times$ $10^{49} / n_{\mathrm{cm}^{-3}} \mathrm{erg}$. These results fulfill the observational constraints, but the problem remains that the proton spectrum is particularly hard.

When we compare the modeling results of both the leptonic and hadronic models, we find that neither can be ruled out. The hadronic model encounters problems by describing the data in a self-consistent way, i.e., it is not possible to describe the radio data with electron and proton spectra with the same spectral index, which would be expected for relativistic particles under the assumption of diffuse shock acceleration theory. Better statistics and possibly more detailed models are needed to solve this question.

Our study of the morphological correlation between radio, $\mathrm{X}$-rays, and $\gamma$-rays has shown (see Sect. 3) that radio and the $\mathrm{X}$-ray emission in the energy range between 0.5 and $1 \mathrm{keV}$ do not coincide with the $\gamma$-ray emission, while the emission in the $\mathrm{X}$-ray regime $2-5 \mathrm{keV}$ shows a correlation with the $\mathrm{TeV} \gamma$-ray emission. This X-ray emission $(2-5 \mathrm{keV})$ is spatially near to a Fe-K-line. Rho et al. (2002) argued that the hard X-ray continuum is synchrotron radiation produced by electrons, which are 


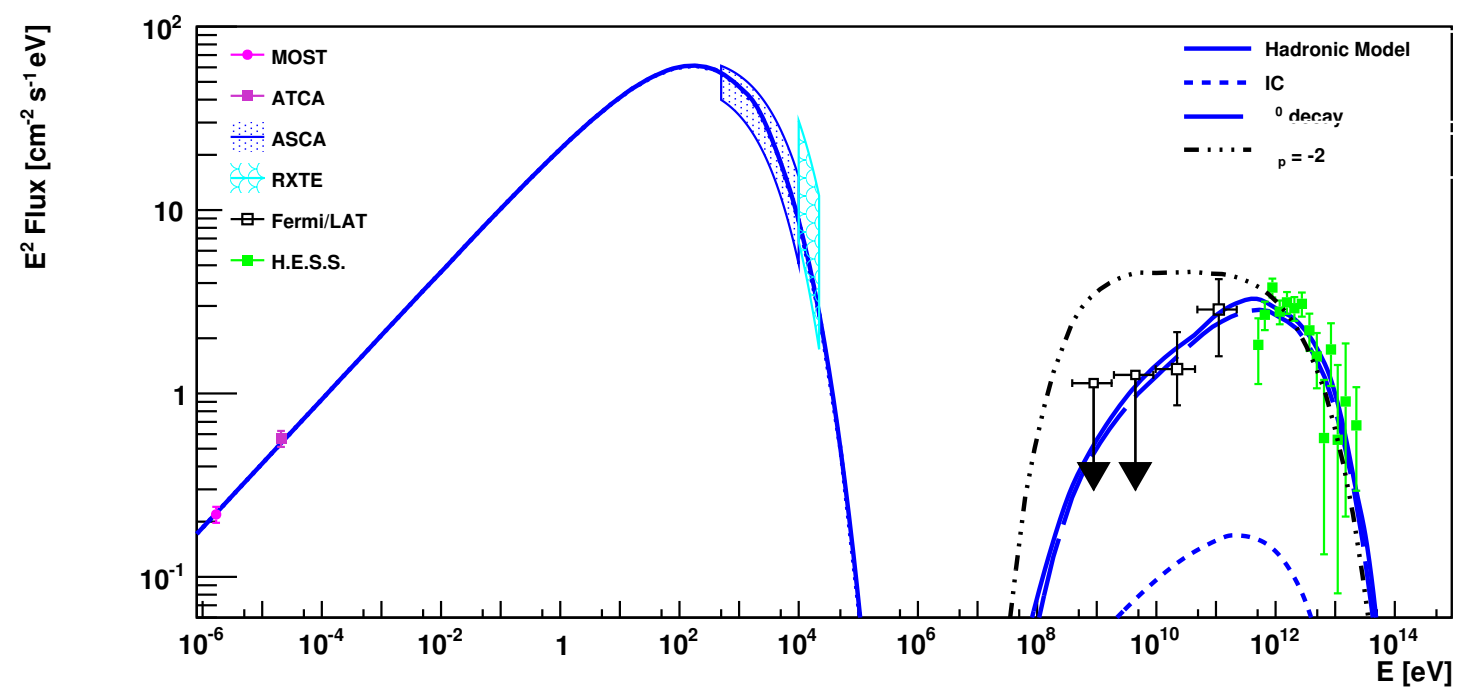

Fig. 5. Spectral energy distribution of RCW 86 for a hadronic scenario. The solid blue lines denote the total broadband emission from the onezone modeling discussed in Sect. 4 The dotted lines show the IC-contributions and the dashed line is that of the $\pi^{0}$-decay contribution. The black dashed dotted line shows the results for a proton spectrum with $\Gamma_{\mathrm{p}}=2$. The radio data points are from Molonglo at $408 \mathrm{MHz}$ and Parkes at $5 \mathrm{GHz}$ (Caswell et al. 1975; Lemoine-Goumard et al. 2012). X-ray data are from ASCA and RXTE from Lemoine-Goumard et al. (2012). The Fermi-LAT data points are taken from Yuan et al. (2014) and the H.E.S.S. data are from this analysis.

accelerated in the reverse shock. It is therefore possible that some of the VHE $\gamma$-ray emission comes from the region shocked by the reverse shock. A possible hint for this is provided by the more centrally filled morphology of the VHE $\gamma$-ray emission with respect to the radio emission, however, the sensitivity of the $\gamma$-ray is not sufficient to draw any firm conclusions about this.

\section{Conclusion}

In this work, we presented the first strong evidence that RCW 86 shows a shell-type morphology in $\mathrm{TeV} \gamma$-rays. The $\mathrm{TeV} \gamma$-ray spectrum favors an exponential cutoff power law with $\Gamma=$ $1.59 \pm 0.22_{\text {stat }} \pm 0.2_{\text {sys }}$ and a cutoff energy $E_{\text {cut }}=3.47 \pm 1.23_{\text {stat }} \pm$ $2.2_{\text {sys }} \mathrm{TeV}$ rather than a pure power law.

The broadband SED can be well described by a simple leptonic one-zone model with a magnetic field strength of $B \approx$ $22 \mu \mathrm{G}$ and $\mathrm{a} \Gamma \approx 2.3$ power-law electron spectrum with an exponential cutoff at $E_{\text {cut }} \approx 19 \mathrm{TeV}$. The kinetic energy of all electrons above $1 \mathrm{GeV}$ is about $0.1 \%$ of the kinetic energy of the supernova explosion of $10^{51} \mathrm{erg}$.

Modeling the broadband data using a hadronic one-zone model requires a hard proton spectrum with index $\Gamma_{\mathrm{p}} \approx 1.7$. This model is incompatible with a conventional $\propto E^{-2}$ acceleration spectrum, but lies between the spectral index value of the test particle approach and the one with strong modified shocks (Malkov 1999; Berezhko \& Ellison 1999). The total energy of all protons above $1 \mathrm{GeV}$ is $W_{\mathrm{p}} \approx 9 \times 10^{49} / n_{\mathrm{cm}^{-3}} \mathrm{erg}$, when a distance of $2.5 \mathrm{kpc}$ are assumed. As a result of limited statistics neither the leptonic nor the hadronic model can be ruled out.

Acknowledgements. The support of the Namibian authorities and of the University of Namibia in facilitating the construction and operation of H.E.S.S. is gratefully acknowledged, as is the support by the German Ministry for Education and Research (BMBF), the Max Planck Society, the German Research Foundation (DFG), the French Ministry for Research, the CNRS-IN2P3 and the Astroparticle Interdisciplinary Programme of the CNRS, the UK Science and Technology Facilities Council (STFC), the IPNP of the Charles University, the Czech Science Foundation, the Polish Ministry of Science and Higher Education, the South African Department of Science and Technology and National Research Founda- tion, and by the University of Namibia. We appreciate the excellent work of the technical support staff in Berlin, Durham, Hamburg, Heidelberg, Palaiseau, Paris, Saclay, and in Namibia in the construction and operation of the equipment. The MOST is operated by The University of Sydney with support from the Australian Research Council and the Science Foundation for Physics within The University of Sydney.

\section{References}

Acero, F., Aharonian, F., Akhperjanian, A. G., et al. 2010, A\&A, 516, A7 Aharonian, F. A. 2013, Astropart. Phys., 43, 71

Aharonian, F., Akhperjanian, A. G., de Almeida, U. B., et al. 2009, ApJ, 692, 1500

Arbutina, B., Urošević, D., Andjelić, M. M., Pavlović, M. Z., \& Vukotić, B. 2012, ApJ, 746, 79

Bamba, A., Koyama, K., \& Tomida, H. 2000, PASJ, 52, 1157

Berezhko, E. G., \& Ellison, D. C. 1999, ApJ, 526, 385

Berge, D., Funk, S., \& Hinton, J. 2007, A\&A, 466, 1219

Bernlöhr, K., Carrol, O., Cornils, R., et al. 2003, Astropart. Phys., 20, 111

Broersen, S., Chiotellis, A., Vink, J., \& Bamba, A. 2014, MNRAS, 441, 3040

Caswell, J. L., Clark, D. H., \& Crawford, D. F. 1975, Aust. J. Phys., 37, 39

de Los Reyes Lopez, R. 2011, in Proc. 32nd Int. Cosmic Ray Conf. (ICRC 2011), 216

de Naurois, M., \& Rolland, L. 2009, Astropart. Phys., 32, 231

Dickel, J. R., Strom, R. G., \& Milne, D. K. 2001, ApJ, 546, 447

Ghavamian, P., Raymond, J., Smith, R. C., \& Hartigan, P. 2001, ApJ, 547, 995

Helder, E. A., Vink, J., Bassa, C. G., et al. 2009, Science, 325, 719

Helder, E. A., Vink, J., Bamba, A., et al. 2013, MNRAS, 435, 910

Hinton, J. A., \& Hofmann, W. 2009, ARA\&A, 47, 523

Kelner, S. R., Aharonian, F. A., \& Bugayov, V. V. 2006, Phys. Rev. D, 74, 034018 Kesteven, M. J., \& Caswell, J. L. 1987, A\&A, 183, 118

Lemoine-Goumard, M., Renaud, M., Vink, J., et al. 2012, A\&A, 545, A28

Long, K. S., \& Blair, W. P. 1990, ApJ, 358, L13

Malkov, M. A. 1999, ApJ, 511, L53

Ohm, S., van Eldik, C., \& Egberts, K. 2009, Astropart. Phys., 31, 383

Pisarski, R. L., Helfand, D. J., \& Kahn, S. M. 1984, ApJ, 277, 710

Porter, T. A., \& Strong, A. W. 2005, Proc. 29th ICRC, 4, 77

Rho, J., Dyer, K. K., Borkowski, K. J., \& Reynolds, S. P. 2002, ApJ, 581, 1116 Smith, R. C. 1997, AJ, 114, 2664

Stephenson, F. R., \& Green, D. A. 2002, Historical supernovae and their remnants, Int. Ser. Astron. Astrophys., vol. 5

Vink, J., Kaastra, J. S., \& Bleeker, J. A. M. 1997, A\&A, 328, 628

Vink, J., Bleeker, J., van der Heyden, K., et al. 2006, ApJ, 648, L33

Völk, H. J., Berezhko, E. G., \& Ksenofontov, L. T. 2005, A\&A, 433, 229

Whiteoak, J. B. Z., \& Green, A. J. 1996, A\&AS, 118, 329 
Williams, B. J., Blair, W. P., Blondin, J. M., et al. 2011, ApJ, 741, 96 Yamaguchi, H., Koyama, K., \& Uchida, H. 2011, PASJ, 63, 837 Yuan, Q., Huang, X., Liu, S., \& Zhang, B. 2014, ApJ, 785, L22

1 Universität Hamburg, Institut für Experimentalphysik, Luruper Chaussee 149, 22761 Hamburg, Germany

2 Max-Planck-Institut für Kernphysik, PO Box 103980, 69029 Heidelberg, Germany

3 Dublin Institute for Advanced Studies, 31 Fitzwilliam Place, Dublin 2, Ireland

4 National Academy of Sciences of the Republic of Armenia, Marshall Baghramian Avenue, 24, 0019 Yerevan, Republic of Armenia

5 Yerevan Physics Institute, 2 Alikhanian Brothers St., 375036 Yerevan, Armenia

6 Institut für Physik, Humboldt-Universität zu Berlin, Newtonstr. 15, 12489 Berlin, Germany

7 University of Namibia, Department of Physics, Private Bag 13301, Windhoek, Namibia

8 GRAPPA, Anton Pannekoek Institute for Astronomy, University of Amsterdam, Science Park 904, 1098 XH Amsterdam, The Netherlands

9 Department of Physics and Electrical Engineering, Linnaeus University, 35195 Växjö, Sweden

${ }^{10}$ Institut für Theoretische Physik, Lehrstuhl IV: Weltraum und Astrophysik, Ruhr-Universität Bochum, 44780 Bochum, Germany

11 GRAPPA, Anton Pannekoek Institute for Astronomy and Institute of High-Energy Physics, University of Amsterdam, Science Park 904, 1098 XH Amsterdam, The Netherlands

12 Institut für Astro- und Teilchenphysik, Leopold-FranzensUniversität Innsbruck, 6020 Innsbruck, Austria

13 School of Chemistry \& Physics, University of Adelaide, 5005 Adelaide, Australia

14 Centre for Space Research, North-West University, 2520 Potchefstroom, South Africa

15 LUTH, Observatoire de Paris, CNRS, Université Paris Diderot, 5 Place Jules Janssen, 92190 Meudon, France

16 LPNHE, Université Pierre et Marie Curie Paris 6, Université Denis Diderot Paris 7, CNRS/IN2P3, 4 Place Jussieu, 75252 Paris, Cedex 5, France

17 Laboratoire Univers et Particules de Montpellier, Université Montpellier 2, CNRS/IN2P3, CC 72, Place Eugène Bataillon, 34095 Montpellier Cedex 5, France

18 DSM/Irfu, CEA Saclay, 91191 Gif-Sur-Yvette Cedex, France

19 Astronomical Observatory, The University of Warsaw, Al. Ujazdowskie 4, 00-478 Warsaw, Poland
20 Aix Marseille Universié, CNRS/IN2P3, CPPM UMR 7346, 13288 Marseille, France

21 Instytut Fizyki Jạdrowej PAN, ul. Radzikowskiego 152, 31-342 Kraków, Poland

22 Funded by EU FP7 Marie Curie, grant agreement No. PIEF-GA2012-332350

23 School of Physics, University of the Witwatersrand, 1 Jan Smuts Avenue, Braamfontein, 2050 Johannesburg, South Africa

24 Laboratoire d'Annecy-le-Vieux de Physique des Particules, Université Savoie Mont-Blanc, CNRS/IN2P3, 74941 Annecy-le-Vieux, France

25 Landessternwarte, Universität Heidelberg, Königstuhl, 69117 Heidelberg, Germany

26 Université Bordeaux, CNRS/IN2P3, Centre d'Études Nucléaires de Bordeaux Gradignan, 33175 Gradignan, France

27 Oskar Klein Centre, Department of Physics, Stockholm University, Albanova University Center, 10691 Stockholm, Sweden

28 Wallenberg Academy Fellow

29 Institut für Astronomie und Astrophysik, Universität Tübingen, Sand 1, 72076 Tübingen, Germany

${ }^{30}$ Laboratoire Leprince-Ringuet, École Polytechnique, CNRS/IN2P3, 91128 Palaiseau, France

31 APC, AstroParticule et Cosmologie, Université Paris Diderot, CNRS/IN2P3, CEA/Irfu, Observatoire de Paris, Sorbonne Paris Cité, 10 rue Alice Domon et Léonie Duquet, 75205 Paris Cedex 13, France

32 Univ. Grenoble Alpes, IPAG; CNRS, IPAG, 38000 Grenoble, France

33 Department of Physics and Astronomy, The University of Leicester, University Road, Leicester, LE1 7RH, UK

34 Nicolaus Copernicus Astronomical Center, ul. Bartycka 18, 00-716 Warsaw, Poland

35 Institut für Physik und Astronomie, Universität Potsdam, KarlLiebknecht-Strasse 24/25, 14476 Potsdam, Germany

36 DESY, 15738 Zeuthen, Germany

37 Obserwatorium Astronomiczne, Uniwersytet Jagielloński, ul. Orla 171, 30-244 Kraków, Poland

38 Universität Erlangen-Nürnberg, Physikalisches Institut, ErwinRommel-Str. 1, 91058 Erlangen, Germany

39 Centre for Astronomy, Faculty of Physics, Astronomy and Informatics, Nicolaus Copernicus University, Grudziadzka 5, 87-100 Torun, Poland

40 Department of Physics, University of the Free State, PO Box 339, 9300 Bloemfontein, South Africa

${ }^{41}$ Heisenberg Fellow (DFG), ITA Universität Heidelberg, 69117 Heidelberg, Germany

42 GRAPPA, Institute of High-Energy Physics, University of Amsterdam, Science Park 904, 1098 XH Amsterdam, The Netherlands 